\title{
Dementia Prevention: A Global Challenge in Urgent Need of Solutions
}

\author{
G. Price ${ }^{1}$, C. Udeh-Momoh ${ }^{1}$, M. Kivipelto ${ }^{1,3}$, L.T. Middleton ${ }^{1,2}$

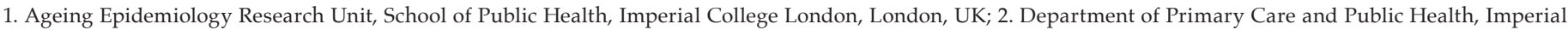

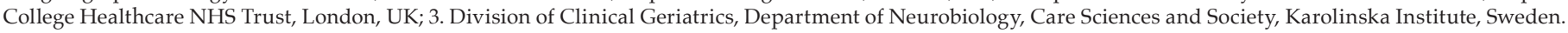

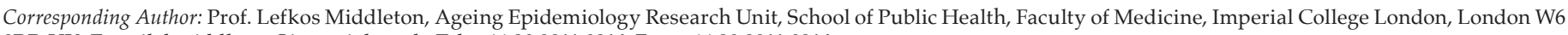
8RP, UK. E-mail: 1.middleton@imperial.ac.uk; Tel: +44 203311 0216; Fax: +44 2033110216.

A n ageing society, whilst representing a major success of humanity in recent decades, brings a significant increase in the numbers of dementia sufferers, world-wide. There is evidence to suggest that successive cohorts remain cognitively healthy for longer than their forebears and reach an older age before developing dementia. This has been attributed to a combination of life-course factors and testifies to the multifactorial aetiology of Alzheimer's disease (AD) and other forms of late-onset dementia (LOD). But as longevity increases, more people live long enough to develop LOD, and survive longer with the condition. Consequently, despite (or because of) improvements in population health, LOD is becoming an increasing burden on society. It is a public health imperative to prevent or delay the onset or clinical progression of this multifactorial disorder. The manuscripts in this issue address a range of aetiological risk factors for LOD, novel technologies and study designs, with implications for preventative strategies.

Age-specific incidence rates for dementia have fallen by an estimated 13\% per decade since 1988 in the United States and Europe (1). Both dementia-free life expectancy and years of survival with dementia have increased (2). Taking into account declining age-specific incidence and increasing life expectancy, the number of cases of dementia is projected to continue to increase over coming decades (3). Reducing the burden of dementia is identified as a key global health priority (4).

The aetiologies of $\mathrm{AD}$ and other $\mathrm{LOD}$ forms are evidently multifactorial, with risk factors identified throughout the life course. The heritability of dementia varies according to type, with that of late-life Alzheimer's disease reaching highly significant levels (h2 ranging between 0.58 and 0.79) (5). Fetal, perinatal and early childhood factors have been proposed as potentially relevant to risk of late-life common and complex diseases, such as dementia or cardiovascular and metabolic conditions (6). Low educational attainment, midand late-life hypertension, diabetes, obesity, physical inactivity, excessive alcohol consumption, smoking, depression, hearing impairment, brain injury, social isolation and air pollution may account collectively for $40 \%$ of dementia cases worldwide (7). Potential explanatory factors which may have contributed to the Received December 20, 2021

Accepted for publication December 24, 2021 reduction in age-adjusted dementia incidence across successive cohorts include higher educational attainment, reductions in smoking and more effective management of cardiovascular diseases and diabetes (8).

With a complex and multifactorial disorder, the identification of aetiological pathways or risk factors should broaden intervention efforts rather than narrowing them. Dementia may in some cases be prevented by interventions which target individual risk factors, as has been demonstrated in the case of risk reduction of cardiovascular diseases via antihypertensive medication (9). But other single-factor strategies have, to date, been less successful. Trials of pharmaceutical interventions targeting beta-amyloid as potential treatments for $\mathrm{AD}$ have yielded predominantly negative results. Some non-pharmaceutical interventions, such as diet, physical exercise and cognitive training, have demonstrated beneficial effects on aspects of cognition though not prevention of dementia (9). Multifactorial aetiology calls for a multidomain approach to intervention, whereby multiple risk factors are addressed concurrently, such as the Finnish Geriatric Intervention Study to prevent cognitive impairment and disability (FINGER) study (10) and the Vascular Care Intervention to Prevent Dementia (preDIVA) study (11). The natural extension of this approach will be the strategy of personalised precision medicine, whereby individuals' personal profiles of risk factors are identified through careful assessment and targeted through bespoke intervention packages delivered to at-risk individuals.

The manuscripts included in this edition relate, in different and complementary ways, to this ambition (12-20). An essential requirement for successful trialling of preventative interventions is the identification and recruitment of at-risk individuals. Mourzi and colleagues present an example of developments in the genetic prediction of the risk and clinical manifestation of dementia in their investigation of the genetics of frailty. Ford et al. discuss the value of neuroimaging in risk prediction and early detection, the pragmatic challenges which limit its widespread adoption at large scale, and the potential for new and emerging technologies to provide solutions to these challenges. Udeh-Momoh et al. present an analysis of the utility and economic viability of plasma amyloid assays in pre-screening for 
AD prevention trials.

Another requirement is the identification and measurement of modifiable risk factors or exposures potentially amenable to intervention. Drawing on preclinical animal models, distinct mechanisms underpinning commonly cited behavioural and nonpharmacological dementia prevention factors with significant potential for AD risk reduction are described comprehensively by Alanko et al. (21). The study by Zheng and colleagues takes forward current knowledge on type 2 diabetes as a risk factor for dementia and identifies a range of comorbidities whose prevention and effective management could contribute to maintaining cognitive health in this at-risk population. The review by Abbott et al. discusses the challenges in the measurement of dietary exposures, and the scope for novel -omics technologies to enhance its accuracy and reliability in investigations of the effects of diet on cognitive health.

The trial protocols in this issue stand as examples of the diversity of approaches, by different means and at different stages of progression to disease, adopted in trials of interventions to prevent or treat dementia. Avgerinos et al. describe a randomized control trial to evaluate the effects of induced ketosis, through the use of ketone monoester, on brain metabolism, cognitive performance and AD pathogenic cascades, in cognitively healthy adults with metabolic syndrome - thus at higher risk for $\mathrm{AD}$. $\mathrm{Xu}$ and colleagues present the Singaporean member of the Worldwide FINGERS international network of multidomain lifestyle interventions to delay cognitive decline in those at risk of dementia. Sindi et al. present the pilot MIND- $\mathrm{AD}_{\mathrm{MINI}}$ randomized trial to extend the FINGER concept to individuals with prodromal AD, with and without medical food supplement. For patients already at the early stages of dementia, Gonzales and colleagues describe a pilot trial of senolytic therapy as a potential modulator of clinical progression.

The manuscripts in this special issue thus argue for diversity - of technologies, risk indicators, therapeutic targets and interventions, both multi-domain and targeted, using a precision medicine model approach - as a fitting and necessary feature of global efforts to reduce the burden of Alzheimer's disease and other forms of late onset dementia.

\section{References}

1. Wolters FJ, Chibnik LB, Waziry R et al. Twenty-seven-year time trends in dementia incidence in Europe and the United States. Neurology 2020; 95 (5) e519-e531. doi: 10.1212/WNL.0000000000010022
2. Grasset L, Pérès $\mathrm{K}$, Joly $\mathrm{P}$ et al. Secular trends of mortality and dementia-free life expectancy over a 10-year period in France. Eur J Epidemiol 2019; 34, 115-123. doi: 10.1007/s10654-019-00482-w

3. Ahmadi-Abhari S, Guzman-Castillo M, Bandosz P et al. Temporal trend in dementia incidence since 2002 and projections for prevalence in England and Wales to 2040: modelling study. BMJ 2017; 358:j2856. doi: 10.1136/ bmj.j2856

4. Shah H, Albanese E, Duggan C et al. Research priorities to reduce the global burden of dementia by 2025. Lancet Neurology 2016; 15 (12), 1285-1294. doi: 10.1016/S1474-4422(16)30235-6

5. Gatz M, Reynolds CA, Fratiglioni L et al. Role of genes and environments for explaining Alzheimer disease. Arch Gen Psychiatry 2006; 63 (2),168-174. doi:10.1001/archpsyc.63.2.168

6. Whalley LJ, Dick FD, \& McNeill G. A life-course approach to the aetiology of late-onset dementias. Lancet Neurology 2006; 5 (1), 87-96. doi: $10.1016 /$ S1474-4422(05)70286-6

7. Livingston G, Huntley J, Sommerlad A, et al. Dementia prevention, intervention, and care: 2020 report of the Lancet Commission. Lancet 2020; 396: 413-446. doi: 10.1016/S0140-6736(20)30367-6

8. Wu Y-T, Beiser AS, Breteler MMB, et al. The changing prevalence and incidence of dementia over time - current evidence. Nat Rev Neurol 2017; 13 (6): 327-339 doi: 10.1038/nrneurol.2017.63

9. Livingston G, Sommerlad A., Orgeta V et al. Dementia prevention, intervention, and care. Lancet 2017; 390: 2673-734. doi: 10.1016/S01406736(17)31363-6

10. Ngandu T, Lehtisalo J, Solomon A, et al. A 2 year multidomain intervention of diet, exercise, cognitive training, and vascular risk monitoring versus control to prevent cognitive decline in at-risk elderly people (FINGER): a randomised controlled trial. Lancet 2015; 385: 2255-2263 doi: 10.1016/S0140-6736(15)604615

11. Moll van Charante EP, Richard E, Eurelings LS et al. Effectiveness of a 6-year multidomain vascular care intervention to prevent dementia (preDIVA): a cluster-randomised controlled trial. Lancet 2016; 388: 797-805, doi: 10.1016/ S0140-6736(16)30950-3

12. Udeh-Momoh C, Zheng B, Sandebring-Matton A et al. Blood Derived Amyloid Biomarkers for Alzheimer's Disease Prevention. J Prev Alz Dis 2022;1(9):12-21, doi: 10.14283 /jpad.2021.70

13. Gonzales MM, Garbarino VR, Marques Zilli E et al. Senolytic Therapy to Modulate the Progression of Alzheimer's Disease (SToMP-AD): A Pilot Clinical Trial. J Prev Alz Dis 2022;1(9):22-29, doi: 10.14283/jpad.2021.62

14. Sindi S, Thunborg C, Rosenberg A et al. Multimodal Preventive Trial for Alzheimer's Disease: MIND-ADmini Pilot Trial Study Design and Progress. J Prev Alz Dis 2022;1(9):30-39, doi: 10.14283/jpad.2022.4

15. Xu X, Chew KA, Wong ZX et al. The SINgapore GERiatric Intervention Study to Reduce Cognitive Decline and Physical Frailty (SINGER): Study Design and Protocol. J Prev Alz Dis 2022;1(9):40-48, doi: 10.14283/jpad.2022.5

16. Abbott KA, Posma JM, Garcia-Perez I et al. Evidence-Based Tools for Dietary Assessments in Nutrition Epidemiology Studies for Dementia Prevention. J Prev Alz Dis 2022;1(9):49-53, doi: 10.14283/jpad.2022.6

17. Avgerinos KI, Mullins RJ, Egan JM et al. Ketone Ester Effects on Biomarkers of Brain Metabolism and Cognitive Performance in Cognitively Intact Adults $\geq$ 55 Years Old. A Study Protocol for a Double-Blinded Randomized Controlled Clinical Trial. J Prev Alz Dis 2022;1(9):54-66, doi: 10.14283/jpad.2022.3

18. Ford J, Kafetsouli D, Wilson $\mathrm{H}$ et al. At a Glance: An Update on Neuroimaging and Retinal Imaging in Alzheimer's Disease and Related Research. J Prev Alz Dis 2022;1(9):67-76, doi: 10.14283/jpad.2022.7

19. Mourtzi N, Hatzimanolis A, Xiromerisiou G et al. Association between 9p21-23 Locus and Frailty in a Community-Dwelling Greek Population: Results from the Hellenic Longitudinal Investigation of Ageing and Diet. J Prev Alz Dis 2022;1(9):77-85, doi: 10.14283/jpad.2022.2

20. Zheng B, Su B, Udeh-Momoh C et al. Associations of Cardiovascular and NonCardiovascular Comorbidities with Dementia Risk in Patients with Diabetes: Results from a Large UK Cohort Study. J Prev Alz Dis 2022;1(9):86-91, doi: 10.14283 /jpad.2022.8

21. Alanko V, Udeh-Momoh C, Kivipelto M et al. Mechanisms Underlying NonPharmacological Dementia Prevention Strategies: A Translational Perspective. J Prev Alz Dis 2022;1(9):3-11, doi:10.14283/jpad.2022.9

How to cite this article: G. Price, C. Udeh-Momoh, M. Kivipelto, et al. Dementia Prevention: A Global Challenge in Urgent Need of Solutions. J Prev Alz Dis 2022;1(9):1-2, http:/ / dx.doi.org/10.14283/jpad.2022.10 